\title{
Followup imaging studies in metastatic castration-resistant prostate cancer: An individualized approach
}

\author{
Myuran Thana, MD; Lori A. Wood, MD
}

Queen Elizabeth II Health Sciences Centre, Halifax, NS, Canada

Cite as: Can Urol Assoc J 2019;13(6):201-2. hitp://dx.doi.org/10.5489/cuaj.6033

See related article on page 192

$\mathrm{T}$ he development of castration resistance in metastatic prostate cancer has generally been considered one of the terminal phases of the disease. However, with advancements in systemic therapy, men with metastatic castration-resistant prostate cancer (mCRPC) can have relatively long survival and good quality of life. With first-line treatment using newer androgen receptor axis-targeted medications, such as abiraterone acetate or enzalutamide, median overall survival approaches three years, with a radiographic progression-free survival (RPFS) of 16.5-20 months. ${ }^{1,2}$

With prolonged periods of disease stability, the question of how best to follow these patients is an important one with no clear answer. While routine prostate-specific antigen (PSA) testing is inexpensive and relatively straightforward for patients to undergo on a regular basis, scheduled imaging studies with computed tomography (CT) and bone scans can be more involved, burdensome, and costly. However, imaging studies are much more useful in documenting the extent of disease progression and for identifying sites responsible for patient symptoms. Further, a subset of mCRPC patients may have radiographic progression in the absence of PSA progression. ${ }^{3}$

In this edition of the Canadian Urological Association Journal, Martin et al outline the results of their analysis of patients treated on the COU-AA-302 clinical trial who received first-line abiraterone acetate plus prednisone (AA-P) for $\mathrm{mCRPC}$ to determine factors associated with RPFS. ${ }^{4}$ Using these factors, patients were divided into groups at high and low risk of RPFS. Their data show that among the $32 \%$ of patients with bone-only disease and $\mathrm{a} \geq 50 \%$ drop in PSA after eight weeks of treatment, the risk of RPFS was low, only 93\% at six months and $80 \%$ at one year. In contrast, patients with bone and soft tissue disease, as well as a $<50 \%$ reduction in baseline PSA at eight weeks (15\% of the total patients) were at a much higher risk for progression, with $55 \%$ and $34 \%$ RPFS at six months and 12 months, respectively.

Using these readily available parameters, the authors suggest that scheduled surveillance imaging can be used less fre-

quently in the low-risk group in view of their long RPFS. This suggestion is limited by a lack of prospective validation and whether these data also apply to patients receiving first-line treatment with agents other than AA-P, those with visceral organ metastasis (who were excluded from the COU-AA-302 trial), or to patients receiving later lines of therapy.

To date, there is a lack of evidence to guide clinicians in determining the role for routine imaging in the absence of PSA or clinical (i.e., symptomatic) progression. As such, guidelines related to this matter vary considerably in their recommendations. In their most recent guidelines, the National Comprehensive Cancer Network, ${ }^{5}$ European Association of Urology, ${ }^{6}$ and European Society of Medical Oncology ${ }^{7}$ all recommend routine surveillance imaging (at variable intervals, generally every 3-6 months) in mCRPC. In contrast, a recent American Society of Clinical Oncology provisional clinical opinion recommended against routine imaging (except in cases where PSA monitoring is felt to be unreliable). ${ }^{8}$ The most recent Canadian Urological Association guideline did not specifically comment on surveillance imaging in this patient population. ${ }^{9}$ Understandably, there is also considerable variation in clinical practice. At a recent international conference, while a majority of attendees favoured routine imaging, 44\% were in favour of pursuing imaging only in response to a rising PSA or clinical symptoms. ${ }^{10}$

Thus, the results presented by Martin et al provide a basis for an individualized approach based on the presence or absence of predictive risk factors. It is important to consider, however, other factors that may not have been captured in this dataset, derived from a randomized control trial of relatively well patients with good performance status. Other baseline factors, including PSA doubling time and duration of hormone sensitivity, could possibly be important but were not analyzed in this study. As well, in real-world practice, many mCRPC patients are elderly, frail, and have multiple comorbidities. Some of these patients may not be ideal candidates for subsequent lines of therapy and the decision to switch from a relatively well-tolerated first-line option may be strongly influenced by the patient's symptoms and clinical status as opposed to radiographic progression alone.

Routine surveillance imaging should be considered similar to other interventions in medicine, with benefits (early 
Thana et al

detection of progressive disease, identification of symptomatic sites for local therapy) and risks (false positive results, early discontinuation of therapy, complications from radiocontrast administration). There are also significant costs associated with cross-sectional imaging; using these resources appropriately, including avoiding them when feasible and appropriate, may be prudent from both an economical and patient management standpoint. Therefore, the potential need for regularly scheduled imaging should be balanced with the risk of disease progression on an individualized basis. Using clinical factors to identify "bad players" at high risk of early progression may help tip the scales in opting for closer followup and routine imaging as an appropriate strategy for those patients, while foregoing scheduled imaging in those in the lowest-risk group. Ultimately, involving patients themselves in an educated discussion to come to a shared decision may be a valuable approach.

Competing interests: Dr. Wood has been an advisory board member (with no financial compensation) for Astellas, Novartis, and Pfizer; and has participated in clinical trials supported by Aragon, AstraZeneca, BMS, Exelixis, Merck, Pfizer, and Roche. Dr. Thana reports no competing personal or financial interests.

\section{References}

1. Ryan CJ, Smith MR, Fizazi K, et al. Abiraterone acetate plus prednisone vs. placebo plus prednisone in chemotherapy-naive men with metastatic castration-resistant prostate cancer (COU-AA-302): Final overall survival analysis of a randomized, double-blind, placebo-controlled phase 3 study. Lancet Oncol 2015;16:152-60. https://doi.org/10.1016/S1470-2045(14)71205-7

2. Beer $T M$, Armstrong $A J$, Rathkopf $D$, et al. Enzalutamide in men with chemotherapy-naive metastatic castration-resistant prostate cancer: Extended analysis of the phase 3 PREVAll study. Eur Urol 2017;71:151-4. https://doi.org/10.1016/i.eururo.2016.07.032

3. Bryce AH, Alumkal JJ, Armstrong A, et al. Radiographic progression with non-rising PSA in metastatic castration-resistant prostate cancer: Post-hoc analysis of PREVAlL. Prostate Cancer Prostatic Dis 2017;20:221-7. https://doi.org/10.1038/pcan.2016.71

4. Martin $\amalg$, Alibhai SMH, Komisarenko M, et al. Identification of subgroups of metastatic castrate-resistant prostate cancer (mCRPC) patients treated with abiraterone plus prednisone at low- vs. high-risk of radiographic progression: An analysis of COU-AA-302. Can Urol Assoc J 2018 [Epub 2018 Nov 5]. https://doi.org/10.5489/cuaj.5586

5. National Comprehensive Cancer Network. Prostate Cancer (Version 2.2019). Available at: http://www. nccn.org/professionals/physician_gls/pdf/prostate.pdf. Accessed April 16, 2019.

6. Cornford P, Bellmunt J, Bolla M, et al. EAU-ESTRO-SIOG guidelines on prostate cancer. Part Il: Treatment of relapsing, metastatic, and castration-resistant prostate cancer. Eur Urol 2017;71:630-42. hittps://doi.org/10.1016/i.eururo.2016.08.002

7. Parker C, Gillessen S, Heidenreich A, et al. Cancer of the prostate: ESMO clinical practice guidelines for diagnosis, treatment and followup. Ann Oncol 2015;26:v69-77. https://doi.org/10.1093/annonc/mdv222

8. Virgo KS, Basch E, Loblaw DA, et al. Second-line hormonal therapy for men with chemotherapy-naive, castration-resistant prostate cancer: American Society of Clinical Oncology provisional clinical opinion. J Clin Oncol 2017;35:1952-64. https://doi.org/10.1200/JC0.2017.72.8030

9. Saad F, Chi K, Finelli A, et al. The 2015 CUA-CUOG guidelines for the management of castration-resistant prostate cancer (CRPC). Can Urol Assoc J 2015;9:90-6. https://doi.org/10.5489/cuai.2526

10. Gillessen S, Attard G, Beer TM, et al. Management of patients with advanced prostate cancer: The report of the Advanced Prostate Cancer Consensus Conference APCCC 2017. Eur Urol 2018;73:178-211. https:// doi.org/10.1016/j.eururo.2017.06.002

Correspondence: Dr. Myuran Thana, Queen Elizabeth II Health Sciences Centre, Halifax, NS, Canada; mthana@dal.ca 